\title{
Clinical and Radiographic Oral Manifestations in Pediatric Patients with Chronic Renal Failure on Dialysis Therapy
}

\author{
Rivas $\mathrm{UO}^{1 *}$, Loza $\mathrm{R}^{2 *}$, Sacsaquispe $\mathrm{SJ}^{1}$ and \\ Calderón $\mathrm{V}^{3}$ \\ ${ }^{1}$ Department of Medicine, Surgery and Oral Pathology, \\ Faculty of Dentistry, Cayetano Heredia University, Perú \\ ${ }^{2}$ Pediatric Nephrology Unit, Cayetano Heredia Hospital, \\ Cayetano Heredia University, Perú \\ ${ }^{3}$ Department of Oral and Maxillofacial Radiology, Faculty \\ of Dentistry, Cayetano Heredia University, Perú \\ *Corresponding author: Rivas UO, Department \\ of Medicine, Surgery and Oral Pathology, Faculty of \\ Dentistry, Cayetano Heredia University, Urb Ingeniería \\ Lima, Perú
}

Loza R, Pediatric Nephrology Unit, Cayetano Heredia Hospital Lima, Urb Ingeniería, Lima, Perú

Received: September 19, 2018; Accepted: October 23, 2018; Published: October 30, 2018

\begin{abstract}
Objective: To describe clinical and radiological oral manifestations in children with chronic renal failure on dialysis therapy and the relationship with disease, period of dialysis, and history of secondary hyperparathyroidism.

Methods: A comparative study of 2 independent groups, including thirtythree children with chronic renal failure on dialysis (between 7-16 years old) and 33 children without chronic renal failure and with similar characteristics was conducted. Intraoral examinations, including a smear of oral mucosa and digital panoramic maxillary radiographs, were performed.
\end{abstract}

Results: Among children with chronic renal failure, 16 (48.5\%) had xerostomia, $18(54.5 \%)$ had uremic breath, $16(48.5 \%)$ showed changes in oral mucosa, $24(72.7 \%)$ had regular oral hygiene with a low rate of dental caries, 20 (60.6\%) had enamel hypoplasia, 25 (75.8\%) tested positive for Candida hyphae, $5(15.2 \%)$ had changes in the shape of the mandibular cortex, and $14(42.4 \%)$ had signs of osteopenia in the jaws.

Conclusion: We found significant differences between the groups with and without chronic renal failure for xerostomia, uremic breath, changes in oral mucosa, enamel hypoplasia, dental caries, mandibular cortical shape, and signs of osteopenia. Moreover, we observed significant relationships between several aforementioned findings and the period of kidney disease, duration of dialysis, and presence of osteodystrophy.

Keywords: Chronic renal failure; Oral manifestations; Dental digital radiography; Dialysis; Secondary hyperpara thyroidism

\section{Introduction}

Children with Chronic Renal Failure (CRF) may exhibit oral conditions of interest, such as xerostomia, because both stimulated and unstimulated salivary flow rates decrease in patients on hemodialysis compared with those in healthy individuals $[1,2]$.

Excess urea and other nitrogen blood products cause some ammoniacal products to appear in body fluids, including saliva. This issue produces dysgeusia in the mouth, characterized by a salty metallic taste and uremic halitosis [3]. Therefore, salivary $\mathrm{pH}$ is significantly more alkaline, which could explain the reduced susceptibility of children with CRF to dental caries [4]. Oral ulcers may appear in mucosa weakened by uremia or anemia, making them more sensitive to physical irritation $[5,6]$. Hematologic and vascular changes occur, with a number of local changes such as paleness, appearance of petechiae or ecchymosis, and gingival bleeding. Compared with healthy individuals, children with uremia may have a reduced gingival inflammatory response to bacterial plaque. Therefore, patients with CRF are predisposed to a greater accumulation of plaque and elevated calculus formation $[7,8]$.

Enamel hypoplasia also occurs in children with CRF because damage to tooth development may result from metabolic problems associated with CRF and may occur following the diagnosis of kidney disease [9]. Secondary hyperparathyroidism, which often develops in patients with renal failure, can cause several changes in the jaw, such as increased marrow spaces, decreased trabeculae, decreased mandibular cortical thickness, total or partial loss of alveolar lamina dura, a widened periodontal ligament space, lytic areas, and abnormal bone scarring after tooth extraction [10]. There is a positive relationship between the effect of the duration of dialysis and oral health parameters-the plaque index, gingival index, periodontal attachment loss, and probing depth-i.e., the state of dental and periodontal health worsens with more time on dialysis in patients with CRF [11].

Moreover, the duration of renal disease and duration of dialysis are related to the degree of enamel hypoplasia and dental plaque index, demonstrating the influence of these factors on the oral condition of patients [12].

The aim of this research was to describe the clinical and radiological oral manifestations of pediatric patients with and without CRF by determining the relationship of such manifestations with the duration of disease and renal therapy, as well as with a history of secondary hyperparathyroidism. 
Table 1: Clinical oral manifestations observed between patients with and without CRF.

\begin{tabular}{|c|c|c|c|c|c|c|c|c|}
\hline \multirow[b]{3}{*}{ Manifestation } & \multirow[b]{3}{*}{ Categories } & \multicolumn{4}{|c|}{ GROUP } & \multirow{2}{*}{\multicolumn{2}{|c|}{ Total }} & \multirow{3}{*}{$\mathrm{p}+$} \\
\hline & & \multicolumn{2}{|c|}{ With CRF $(n=33)$} & \multicolumn{2}{|c|}{ Without CRF $(n=33)$} & & & \\
\hline & & $\mathrm{n}$ & $\%$ & $\mathrm{n}$ & $\%$ & $\mathrm{n}$ & $\%$ & \\
\hline \multirow[t]{2}{*}{ Xerostomia } & No & 17 & 51.5 & 32 & 97 & 49 & 74.2 & $0.000^{*}$ \\
\hline & Yes & 16 & 48.5 & 1 & 3 & 17 & 25.8 & \\
\hline \multirow[t]{2}{*}{ Uremic breath } & No & 15 & 45.5 & 33 & 100 & 48 & 72.7 & $0.000^{*}$ \\
\hline & Yes & 18 & 54.5 & 0 & 0 & 18 & 27.3 & \\
\hline \multirow[t]{4}{*}{ Alterations in oral mucosa } & No & 17 & 51.5 & 33 & 100 & 50 & 75.8 & $0.000^{*}$ \\
\hline & Pallor & 14 & 42.4 & 0 & 0 & 14 & 21.2 & \\
\hline & Petechiae & 1 & 3 & 0 & 0 & 1 & 1.5 & \\
\hline & Gingival bleeding & 1 & 3 & 0 & 0 & 1 & 1.5 & \\
\hline \multirow[t]{2}{*}{ Oral Hygiene Index } & Good & 9 & 27.3 & 15 & 45.5 & 24 & 36.4 & 0.13 \\
\hline & Regular & 24 & 72.7 & 18 & 54.5 & 42 & 63.6 & \\
\hline \multirow[t]{7}{*}{ Enamel hypoplasia } & No & 13 & 39.4 & 33 & 100 & 46 & 69.7 & $0.000^{*}$ \\
\hline & $A$ & 4 & 12.1 & 0 & 0 & 4 & 6.1 & \\
\hline & B & 8 & 24.2 & 0 & 0 & 8 & 12.1 & \\
\hline & $\mathrm{C}$ & 2 & 6,1 & 0 & 0 & 2 & 3 & \\
\hline & $\mathrm{D}$ & 2 & 6.1 & 0 & 0 & 2 & 3 & \\
\hline & $E$ & 1 & 3 & 0 & 0 & 1 & 1.5 & \\
\hline & $\mathrm{F}$ & 3 & 9,1 & 0 & 0 & 3 & 4.5 & \\
\hline \multirow[t]{2}{*}{ Oral candidiasis } & Negative & 25 & 75.8 & 28 & 84.8 & 53 & 80.3 & 0.35 \\
\hline & Positive & 8 & 24.2 & 5 & 15.2 & 13 & 19.7 & \\
\hline
\end{tabular}

${ }^{*} \mathrm{p}<0.05$ significant, + Chi squared

\section{Materials and Methods}

This study is a cross-sectional analysis of two independent groups. Children with CRF came from the Pediatric Nephrology Unit of Cayetano Heredia National Hospital, Lima-Peru. Controls attended the Department of Oral and Maxillofacial Radiology, Faculty of Dentistry, of Cayetano Heredia University (CHU), Lima-Peru. The protocol was approved by the CHU Ethics Committee. Parents and patients gave written informed consent to participate.

The inclusion criteria used to select patients were under 18 years of age and the presence of the 7 permanent lower left teeth visible in a radiograph.

The same dentist carried out the oral examinations and took smears of oral mucosa. An oral and maxillofacial pathologist at the Department of Medicine, Surgery and Oral Pathology, CHU, was responsible for reading and interpreting the smears of oral mucosa. The radiological interpretation was conducted by an oral and maxillofacial radiologist of the Department of Oral and Maxillofacial Radiology, CHU. The following oral examination data were collected: xerostomia using a test palette (criterion used at the Department of Medicine, Surgery and Oral Pathology, CHU), uremic breath determined by detecting a urine smell on the patient's breath by asking the patient to exhale air through the mouth, and the presence of alterations in oral mucosa. For the accumulation of dental plaque, we used Greene and Vermillion's Oral Hygiene Simplified Index (OHI-s) [13]. We recorded the number of locations with decayed, missing, and filled teeth, both permanent and deciduous (DMFT and/ or dmft used, respectively), according to the index. We also used the Jackson-Al-Alousi Index [14] for enamel hypoplasia.

Smears were performed in oral mucosa (dorsum of the tongue or buccal mucosa) using a Periodic Acid-Schiff (PAS) reagent to determine the presence of Candida hyphae.

Panoramic radiographs of the jaws were taken on a digital panoramic unit, a Sirona Orthophos XG3 ${ }^{\circ}$. The radiographs were labeled in the database system; the radiologist who made the observations (in a dark environment) was blinded to patient identity and age.

The following digital panoramic radiograph data were collected:

Radiographic signs of osteopenia (trabeculae in the jaw, loss of lamina dura and alveolar bone lytic areas) and mandibular cortical shape assessed by the mandibular cortical index according to Klemetti [15].

The results obtained in this study were analyzed using SPSS version 20. The Chi square test was used to compare the clinical and radiological oral manifestations observed between the study and control groups. Mann-Whitney U, Kruskal-Wallis and one-way ANOVA tests were used to determine the relationship between the duration of illness and duration of renal therapy in patients with clinical and radiological oral manifestations. The Chi square test was used to determine the relationship between the history of secondary hyperparathyroidism and any radiological manifestations. Statistical significance was set at a value of $\mathrm{p}<0.05$. 
Table 2: Radiological oral manifestations observed between patients with and without CRF.

\begin{tabular}{|c|c|c|c|c|c|c|c|c|}
\hline \multirow[b]{3}{*}{ Manifestations } & \multirow[b]{3}{*}{ Categories } & \multicolumn{4}{|c|}{ GROUP } & \multirow{2}{*}{\multicolumn{2}{|c|}{ Total }} & \multirow{3}{*}{$\mathrm{p}+$} \\
\hline & & \multicolumn{2}{|c|}{ With CRF $(n=33)$} & \multicolumn{2}{|c|}{ Without CRF $(n=33)$} & & & \\
\hline & & $\mathrm{n}$ & $\%$ & $\mathrm{n}$ & $\%$ & $\mathrm{n}$ & $\%$ & \\
\hline & C1 & 28 & 84.8 & 33 & 100 & 61 & 92.4 & 0.06 \\
\hline Form of mandibular cortex* & $\mathrm{C} 2$ & 3 & 9.1 & 0 & 0 & 3 & 4.5 & \\
\hline \multirow[t]{2}{*}{ Uremic breath } & $\mathrm{C} 3$ & 2 & 6.1 & 0 & 0 & 2 & 3 & \\
\hline & Yes & 18 & 54.5 & 0 & 0 & 18 & 27.3 & \\
\hline \multirow[t]{4}{*}{ Radiological signs of osteopenia in the jaw } & None & 19 & 57.6 & 31 & 93.9 & 50 & 75.8 & $0.000^{* *}$ \\
\hline & Reduced trabeculation & 0 & 0 & 2 & 6.1 & 2 & 3 & \\
\hline & Loss of alveolar hard film & 10 & 30.3 & 0 & 0 & 10 & 15.2 & \\
\hline & Lithic areas in the bone & 4 & 12.1 & 0 & 0 & 4 & 6.1 & \\
\hline
\end{tabular}

*mandibular cortical index by Klemetti

${ }^{* *} p<0.05$ significant, + Chi squared.

\section{Results}

A total of 33 CRF patients received hemodialysis therapy (42.1\%) and peritoneal dialysis (57.9\%), including 17 females and 16 males with an average age $\dot{X}=152.88 \pm 30.62$ months. In the CRF group, $57.6 \%$ of patients had a history of secondary hyperparathyroidism, the duration of renal disease was $\dot{X}=46.64 \pm 43.92$ months, and the duration of renal therapy was $\dot{X}=21.94 \pm 19.28$ months. There were 33 patients without CRF, including 19 females and 14 males with an average age $\dot{X}=145.12 \pm 35.34$ months.

The groups with and without CRF exhibited significant differences in xerostomia $(p=0.000)$, uremic breath $(p=0.000)$, alterations in the oral mucosa $(\mathrm{p}=0.000)$, and enamel hypoplasia $(\mathrm{p}=0.000)($ Table 1$)$.

No significant differences were observed for the oral hygiene index and oral candidiasis. Considering only the hardened plaque component of the oral hygiene index, $78.8 \%$ of patients with CRF and $12.12 \%$ of patients without CRF had calculus deposits. In the group with CRF, the DMFT index and/or dmft was 2.58, indicating a low rate of decay. In the group without CRF, this index was 4.55 , indicating a high rate of caries.

Radiographic evidence of osteopenia $(\mathrm{p}=0.000)$ was significantly different between the group with CRF and the group without CRF except for the form of the mandibular cortex. However, when we analyzed by considering only "altered" versus "unaltered" mandibular cortex, there were significant differences $(\mathrm{p}=0.020)$ between the two groups (Table 2).

The duration of kidney disease was directly related to radiological signs of osteopenia $(\mathrm{p}=0.047)$. We observed a direct relationship of the duration of kidney disease with alterations of the oral mucosa and enamel hypoplasia. However, when we analyzed these relationships as "presence" versus "absence", $\mathrm{p}=0.000$. The same pattern was observed for the variable shape of the cortex regardless of the category (only "altered" and "altered"), which was directly related to the duration of kidney disease, $\mathrm{p}=0.007$.

The duration of renal therapy was directly related to variable alterations in the oral mucosa and enamel hypoplasia (only "presence" and "absence"), p=0.000. The same pattern was observed for the variable form of the mandibular cortex and radiographic signs
Table 3: Duration of renal disease and duration of renal therapy in relation to clinical and radiological manifestations in patients with CRF.

\begin{tabular}{|c|c|c|}
\hline & $\begin{array}{c}\text { Duration of renal } \\
\text { disease }\end{array}$ & $\begin{array}{c}\text { Duration of renal } \\
\text { therapy }\end{array}$ \\
\hline Buccal Manifestations & p value & p value \\
\hline Xerostomia & $0.505^{\star}$ & $0.234^{\star}$ \\
\hline Uremic breath & $0.718^{\star}$ & $0.246^{\star}$ \\
\hline Alterations in oral mucosa & $0.905^{\star}$ & $0.937^{\star}$ \\
\hline Oral Hygiene Index & $0.984^{\star}$ & $0.209^{\star}$ \\
\hline Enamel hypoplasia & $0.051 \dagger$ & $0.167^{\circ}$ \\
\hline Oral candidiasis & $0.817^{\star}$ & $0.933^{\star}$ \\
\hline $\begin{array}{c}\text { Form of the mandibular } \\
\text { cortex }\end{array}$ & $0.527 \dagger$ & $0.281 \dagger$ \\
\hline $\begin{array}{c}\text { Radiological signs of } \\
\text { osteopenia }\end{array}$ & $0.047 \dagger$ & $0.967 \dagger$ \\
\hline
\end{tabular}

Mann-Whitney $\cup$ Test

† Kruskal-Wallis Test

One-way ANOVA test

of osteopenia (only "altered" and "altered"); these variables were directly related to the duration of renal therapy, $\mathrm{p}=0.001$ and $\mathrm{p}=0.000$, respectively.

We detected a direct relationship between a history of secondary hyperparathyroidism and radiological signs of osteopenia, $\mathrm{p}=0.03$ (Table 3).

\section{Discussion}

Significant differences were observed in xerostomia between children with and without CRF. Martins et al. [16] observed a decrease in the salivary flow rate in children with CRF compared with that in controls. However, Al-Nowaiser et al. [11], who analyzed samples of saliva collected from children with CRF, found that the salivary flow rate was similar to that of healthy children.

For the variable uremic breath, significant differences were also found between children with and without CRF. Higher levels of salivary urea have been found in children with CRF [1].

Proctor et al. evaluated the oral mucosa of adult patients with CRF, finding lichen planus and oral hairy leukoplakia, geographic tongue and black hairy tongue. In our investigation, we found alterations in the oral mucosa of children with CRF compared with that of children 


\section{without CRF [5].}

An increased prevalence of enamel hypoplasia has been reported in patients with CRF $[12,17]$. Coincidently, in the present study, we found significant differences between patients with and without CRF. Our study contradicts the findings by Martins et al. [16], who did not find a higher prevalence of enamel hypoplasia in children with $\mathrm{CRF}$ than in controls. Hypoplastic areas in permanent teeth often correspond to the age of onset of CRF (4). Enamel alterations range from white spots to morphological abnormalities. The JacksonAl-Alousi index [14] of enamel hypoplasia was used in another investigation in children with CRF, and $38.09 \%$ of the population had enamel hypoplasia [18]. In our study, $60.6 \%$ of children with CRF showed enamel hypoplasia; the percentage for children without CRF was zero.

The changes in oral health status associated with dental plaque in children with CRF have been examined by many studies. Cengiz et al. [11], Al-Nowaiser et al. [1] and Martins et al. [16] reported increased plaque and calculus deposits in patients with CRF. In the present study, we found no significant differences between children with and without CRF in relation to the oral hygiene index; however, differences between the groups were found by observing only the component calculation. In our study, we found a low incidence of dental caries in children with CRF and a high incidence of dental caries in children without CRF, consistent with previous studies [12,16-19] in which a low incidence of dental caries was observed in subjects with CRF. This finding could be attributed to elevated urea levels in the saliva of patients with CRF, leading to an increase in buffering capacity due to the high ammonia concentration. Increased buffering capacity promotes an alkaline medium, protecting the teeth from acid attack $[1,20]$.

Al-Nowaiser et al. [1] made smears at different sites of the oral mucosa and used Calgon-Ringer solution; the authors found Candida albicans in 11 children in the control group but not in children in the CRF group. Although CRF patients are predisposed to xerostomia, no significant differences were found between children with and without CRF regarding oral candidiasis using PAS cytological examination in the present study. Additionally, in the present investigation, we detected Candida hyphae in children in the control group with clinically healthy oral mucosa.

Buhlin et al. [21] and Frankenthal et al. [22] investigated the loss of periodontal attachment and alveolar bone loss in adults with CRF using conventional panoramic radiographs. In this study, digital radiographs were used because they have a lower risk of radiation. More erosion and greater signs of osteopenia were found in children with CRF than in children without CRF. Cengiz et al. [11] and Davidovich et al. [12] conducted research in children and adults, respectively, finding that oral health indices worsen with the duration of dialysis.

In our study, the presence of alterations in the oral mucosa, enamel hypoplasia, mandibular cortical shape and signs of osteopenia were directly related to the duration of kidney disease and renal therapy.

We were unable to confirm the effect of secondary hyperparathyroidism and alveolar bone loss observed in adult patients with CRF [22]. In other studies of secondary hyperparathyroidism and bone disease, parathyroid hormone levels seven times higher than the normal upper range [23] have been observed. In this study, $57 \%$ of children with CRF had secondary hyperparathyroidism, which revealed a direct relationship between the history of secondary hyperparathyroidism and the signs of osteopenia in children with CRF as determined by the loss of lamina dura and alveolar bone lytic areas. The study has limitations regarding the small population of study subjects compared with healthy children but nevertheless it can be analyzed as independent groups.

\section{Conclusion}

We found significant differences between the groups with and without CRF for xerostomia, uremic breath, changes in oral mucosa, enamel hypoplasia, dental caries, mandibular cortical shape, and signs of osteopenia. Moreover, we observed significant relationships between several aforementioned findings and the period of kidney disease, duration of dialysis, and presence of osteodystrophy.

\section{Acknowledgement}

To children on dialysis at the Cayetano Heredia National Hospital Lima Peru.

\section{References}

1. Al Nowaiser A, Roberts GJ, Trompeter RS, Wilson M, Lucas VS. Oral health and oral streptococcal flora of children with chronic renal failure. Pediatr Nephrol. 2003; 18: 39-45

2. Summers S, Tilakaratne WM, Fortune F, Ashman N. Renal disease and the mouth. The American Journal of Medicine. 2007; 120: 568-573.

3. De Rossi, Glick M. Dental considerations for the patient with renal disease receiving hemodialysis. J Am Dent Assoc. 1996; 127: 211-219.

4. Lucas V, Roberts G. Oro-dental health in children with chronic renal failure and after renal transplantation: a clinical review. Pediatr Nephrol. 2005; 20: 1388-1394.

5. Proctor R, Kumar N, Stein A, Moles D, Porter S. Oral and dental aspects of chronic renal failure. J Dent Res. 2005; 84: 199-208.

6. Antoniades DZ, Markopoulos AK, Andreadis D, Balaskas I, Patrikalou E, Grekas D, et al. Ulcerative uremic stomatitis associated with untreated chronic renal failure: Report of a case and review of the literatura. Oral Surg Oral Med Oral Pathol Oral Radiol Endod. 2006; 101: 608-613.

7. Souza CR, Libério SA, Guerra RN, Monteiro S, Silveira EJ, Pereira AL. Evaluación de la condición periodontal de pacientes renales en hemodiálisis. Rev Assoc Med Bras. 2005; 51: 285-289.

8. Bayraktar G, Kurtulus I, Kazancioglu R, Bayramgurler I, Cintan S, Bural C et al. Evaluation of periodontal parameters in patients undergoing peritoneal dialysis or hemodialysis. Oral Diseases. 2008; 14: 185-189.

9. Imirzalioglu P, Onay EO, Agca E, Ogus E. Dental erosion in chronic rena failure. Clin Oral Invest. 2007; 11: 175-180.

10. Goransson L, Bergrem H. Renal transplant and secondary hyperparathyroidism. Scand J Urol Nephrol. 2007; 41: 553-557.

11. Cengiz MI, Sümer $P$, Cengiz $S$, Yavuz $U$. The effect of the duration of the dialysis in hemodialysis patients on dental and periodontal findings. Oral Diseases. 2009; 15: 336-341.

12. Davidovich E, Schwarz Z, Davidovitch M, Eidelman E, Bimstein E. Ora findings and periodontal status in children, adolescents and young adults suffering from renal failure. J Clin Periodontol. 2005; 32: 1076-1082.

13. Greene JC, Vermillion JR. The simplified oral hygiene index. JADA. 1964; 68: 7-13. 
14. Al-Alousi W, Jackson D, Compton G, Jenkins OC. Enamel mottling in a fluoride and in a non-fluoride community. Br Dent J. 1975; 138: 56-60.

15. Klemetti E, Kolmakov S, Kroger H. Pantomography in assessment of the osteoporosis risk group. Scand J Dent res. 1994; 102: 68-72.

16. Martins C, Siqueira WL, Guimarães LS. Oral and salivary flow characteristics of a group of Brazilian children and adolescents with chronic renal failure. Pediatr Nephrol. 2008; 23: 619-624.

17. Nunn JH, Sharp J, Lambert H, Plant ND, Coulthard MG. Oral health in children with renal disease. Pediatr Nephrol. 2000; 14: 997-1001.

18. Ibarra C, Ruiz MS, Fonseca MP, Gutiérrez FJ, Pozos AJ. Enamel Hypoplasia in children with Renal Disease in a Fluoridated Area. J Clin Pediatr Dent. 2007; 31: 274-278.

19. Nakhjavani $Y$, Bayramy A. The dental and oral status of children with chronic renal failure. J Indian Soc Pedod Prev Dent. 2007; 7-9.
20. Castillo A, Mesa F, Liébana J, García-Martinez O, Ruiz S, García-Valdecasas $\mathrm{J}$, et al. Periodontal and oral microbiological status of an adult population undergoing haemodialysis: a cross-sectional study. Oral Diseases. 2007; 13: 198-205.

21. Buhlin K, Barany P, Heimburger O, Stenvinkel P, Gustafsson A. Oral health and pro-inflammatory status in end-stage renal disease patients. Oral Health Prev dent. 2007; 5: 235-244.

22. Frankenthal S, Nakhoul F, Machrei EE, Green J, Ardekum L, Laufer D, et al. The effect of secondary hyperparathyroidism and hemodialysis therapy on alveolar bone and periodontium. J Clin Periodontol. 2002; 29: 479-483.

23. Elder G. Pathogenesis and management of hyperparathyroidism in end-stage renal disease and after renal transplantation. Nephrology. 2001; 6: 155-160.
Austin J Nephrol Hypertens - Volume 5 Issue 1 - 2018

ISSN : 2381-8964 | www.austinpublishinggroup.com

Rivas et al. (C) All rights are reserved
Citation: Rivas UO, Loza R, Sacsaquispe SJ and Calderón V. Clinical and Radiographic Oral Manifestations in Pediatric Patients with Chronic Renal Failure on Dialysis Therapy. Austin J Nephrol Hypertens. 2018; 5(1): 1077. 